\title{
Combustion Phenomena within a RBCC Engine under Scramjet-mode Operation
}

\author{
By Tomoyuki KomUro ${ }^{1)}$, Sadatake TOMIOKA ${ }^{1)}$, Shohei TAKAGI ${ }^{2}$, Masahiro TAKAHASHI ${ }^{1)}$, \\ Shuichi UEDA $^{1)}$ and Katsuhiro ITOH $^{1)}$ \\ 1) JAXA Kakuda Space Center, Kakuda, Japan \\ ${ }^{2)}$ Department of Aerospace Engineering, Tohoku University, Sendai, Japan
}

(Received June 20th, 2013)

\begin{abstract}
Combination of a scramjet (supersonic combustion ramjet) flow-pass with embedded rocket engines (the combined system termed as Rocket Based Combined Cycle engine) are expected to be the most effective propulsion system for Booster stage of space launch vehicles. At hypersonic regime, it will be operated at rather high rocket engine output for final acceleration with some Isp gains due to air-breathing effects. In this regime, attaining thrust at this high-speed regime becomes very difficult, so that parallel injection of the fuel for scramjet combustion is favorable as the momentum of the injection can contribute to the thrust production. Thus, embedded rocket chamber was supposed to the operated as fuel rich gas generator at very high output. This configuration was tested at simulated flight Mach number of 11 at High Enthalpy Shock Tunnel (HIEST) with detonation tube as the source of the simulated rocket exhaust. However, combustion of the residual fuel in the rocket exhaust with airflow could not be attained in the past study, so that modification was carried out. Combustion was attained within the combustor, however, insufficient mixing was found to responsible for the rather low combustion efficiency, finding being from complementary mixing test at a blow-down type tunnel.
\end{abstract}

Key Words: RBCC Engines, Hypersonic Flight Regime, Combustion Enhancement

\section{Nomenclature}

$\begin{array}{ll}M & : \text { Mach number } \\ p, P & : \text { pressure } \\ U, U c & : \text { velocity, convective velocity } \\ X & : \text { streamwise location from rocket base } \\ \phi & : \text { equivalence ratio } \\ \text { bscripts } & \\ 0 & : \text { stagnant } \\ \text { a } & \text { : airflow } \\ \text { i } & : \text { auxiliary fuel } \\ \text { r } & : \text { rocket exhaust, rocket residual fuel } \\ \text { w } & : \text { wall }\end{array}$

\section{Introduction}

Combination of a scramjet (supersonic combustion ramjet) flow-pass with embedded rocket engines (the combined system termed as Rocket Based Combined Cycle engine) are expected to be the most effective propulsion system for booster stage of launch vehicles, and several engine systems were proposed, such as combined types, ${ }^{1-3)}$ and parallel types. ${ }^{4)}$

For the combined type proposed by Japan Aerospace eXprolation Agency (JAXA), ${ }^{1)}$ various operation conditions are required to attain maximum payload for the launch vehicle with the combined cycle engine, i.e., an ejector-jet mode operation at low speed range (M0 3.5) for best initial acceleration with some thrust gain due to air-breathing effects, a ramjet-mode operation (dual-mode combustion in the scramjet flow pass, M3.5 7) with reduced rocket engine output for acceleration with best specific impulse, and a scramjet-mode operation $(M>7)$ with rather high rocket engine output for final acceleration with some Isp gains due to air-breathing effects. The Aerojet company proposed slightly different operation for its RBCC engine system ${ }^{2}$, adding pure scramjet operation (with embedded rocket engine being turned off) during M6 8, and air-augmented rocket operation (equivalent to the scramjet-mode operation in Ref. 1) above M8.

Also there are many proposals on the RBCC engine system, only few were fabricated and tested at wind tunnel facilities, especially in very high speed regime. The JAXA-proposed engine model was tested at various flight $M$ conditions from M0 to M11. The Aerojet-proposed engine model was also tested but at limited flight $\mathrm{M}$ conditions up to M7. ${ }^{5)}$ Test results on other engine models were scarcely available (only public announcement such as Ref. 3).

In the very high speed regime, the incoming airflow has large enthalpy in respect to the available heat release due to combustion. Thus, attaining thrust at this high-speed regime becomes very difficult. In this regime, parallel injection of fuel for scramjet combustion (termed as ramjet-fuel in the present study) is favorable as the momentum of the fuel jet can contribute to the thrust production. In the JAXA-proposed RBCC system, thus, embedded rocket engines were supposed to the operated 


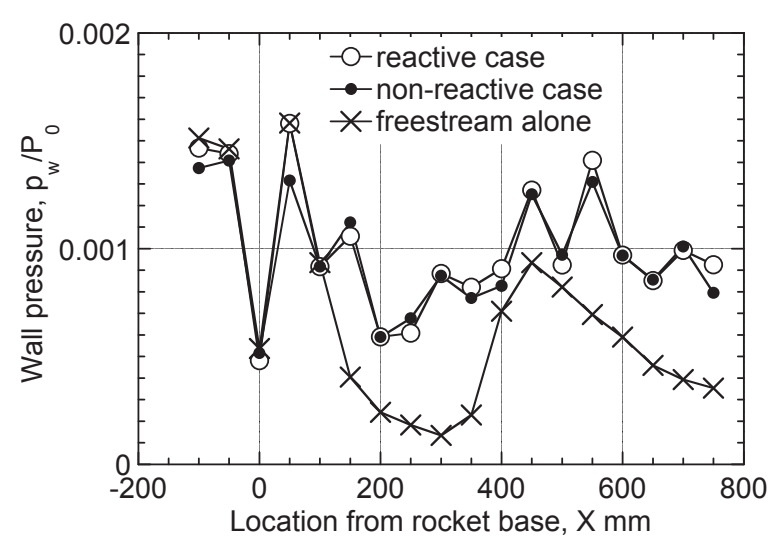

Fig. 1. Wall pressure distributions on cowl wall at Mach $11 \mathrm{flight}$ conditions.

as fuel-rich gas-generators at very high output, so that the ramjet-fuel was included in the fuel-rich rocket exhaust.

This operation mode was tested at JAXA - Kakuda Space Center, using high enthalpy shock tunnel (HIEST) to simulate the hypersonic flight regime (M9 11). The HIEST is a piston-driven shock tunnel facility which is capable to simulate up to Mach 25 enthalpy level (30 $\mathrm{MJ} / \mathrm{kg}$ ) and also capable to select the test medium for reactive case (air) or non-reactive case (nitrogen). The tests, however, showed shortage of combustion of the scramjet-fuel, ${ }^{6)}$ as shown in Fig. 1, i.e., no sizable rise in pressure due to combustion was observed, showing that ignition delay was larger than residence time ( $\mathrm{Da}<<1$ ) and that combustion was rate-controlled by reaction in this condition. Fundamental research on combustion enhancement in this mode through application of so-called auxiliary injection was also conducted, and based on the results, modification of the engine model and operation tested at HIEST was proposed. ${ }^{6}$

In the present study, the modified engine model with auxiliary injector was tested at HIEST, and the results are reported with a focus on combustion phenomena in the engine model. As the very short operation time of HIEST made it difficult to conduct gas sampling to evaluate mixing process within the engine, compliment mixing tests was conducted by matching similarity parameters to those in HIEST tests. These results are also described in the present study.

\section{Engine Tests at HIEST}

An engine model was fabricated and tested at HIEST. Technical challenge for the engine model test was to simulate the rocket plume with combustion gas, which should be ejected during the short duration time of the tunnel operation (typically few milliseconds). For that purpose, a detonation tube was used to supply combustion gas simulating the rocket exhaust. The facility, test model and test method as well as the test results are described in this section

\subsection{Test facility}

The High Enthalpy Shock Tunnel (HIEST, Ref. 7) is the world largest shock tunnel facility, capable to simulate up to Mach 25 enthalpy level $(30 \mathrm{MJ} / \mathrm{kg})$. A piston, driven by high-pressure air, compresses helium within the driver section tube. After rapture of a diaphragm, shock wave travels within the shock-tube section to compress test gas filling the shock-tube section. Either air or nitrogen was used as the test gas to conduct both reactive and non-reactive tests. Table 1 summarized freestream conditions used in the present study.

\begin{tabular}{ccc} 
Table 1. & \multicolumn{3}{c}{ Freestream conditions. } \\
\hline \multirow{2}{*}{$\begin{array}{c}\text { Stagnation } \\
\text { Condition }\end{array}$} & Total temp. & $4550 \mathrm{~K}$ \\
\cline { 2 - 3 } & Total pres. & $17 \mathrm{MPa}$ \\
\cline { 2 - 3 } & Enthalpy & $7.0 \mathrm{MJ} / \mathrm{kg}$ \\
\hline \multirow{3}{*}{$\begin{array}{c}\text { Condition at } \\
\text { nozzle exit }\end{array}$} & Static temp. & 6.7 \\
\cline { 2 - 3 } & Static pres. & $1.9 \mathrm{kPa}$ \\
\cline { 2 - 3 } & Dynamic pres. & $62 \mathrm{kPa}$ \\
\hline
\end{tabular}

\subsection{Engine model, test method and measurements}

The engine model was a rectangular one with a length of $1.45 \mathrm{~m}$, as shown in Fig. 2. This model resembled the engine model (Ref. 8) tested at Ramjet Engine Test Facility (RJTF), with reduced size (about half) and slight differences. A single ramp with angle of 10 degrees, compressed the freestream with a compression ratio of 4.5 , and the airflow pass after compression was $100 \mathrm{~mm}$ in width and $25 \mathrm{~mm}$ in height. Two rocket chambers were installed within an isolator section downstream of the inlet section. The rocket nozzles $(13 \mathrm{~mm}$ in throat diameter and $35 \mathrm{~mm}$ in exit diameter) were located at a backward-facing base ( $40 \mathrm{~mm}$ in height) at the exit of the isolator section. In the present study, streamwise origin $(\mathrm{X}=0)$ was set at the rocket base.

Auxiliary injectors were located at $50 \mathrm{~mm}$ upstream of the rocket base location, on both ramp and cowl walls, while the injection wall were selective (the former termed as ramp-side injection and the latter as cowl-side injection). Eight perpendicular injection orifices (1.5 $\mathrm{mm}$ in diameter) were aligned with even interval in streamwise direction. Room temperature gaseous hydrogen was injected at sonic speed. Fuel equivalence ratio of the auxiliary ramjet-fuel was denoted as $\phi_{i}$.

Downstream of the rocket base, a ramjet-combustor was attached with a diverging angle of 3 degrees on the

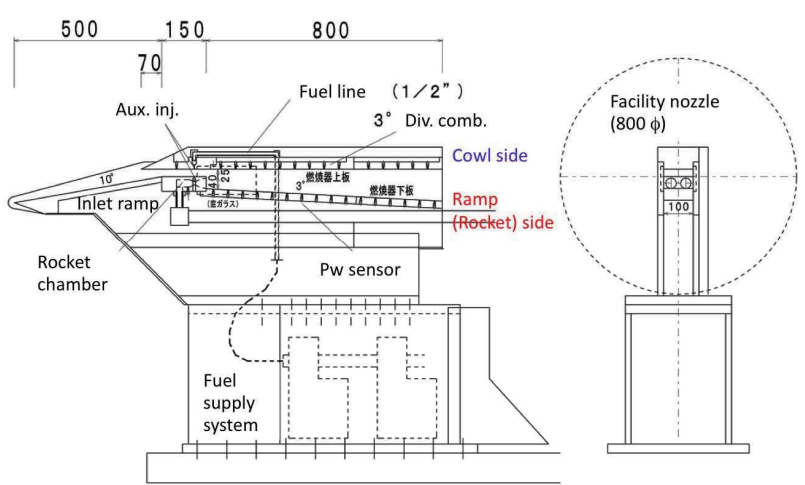

Fig. 2. RBCCengine model testes at HIEST. 


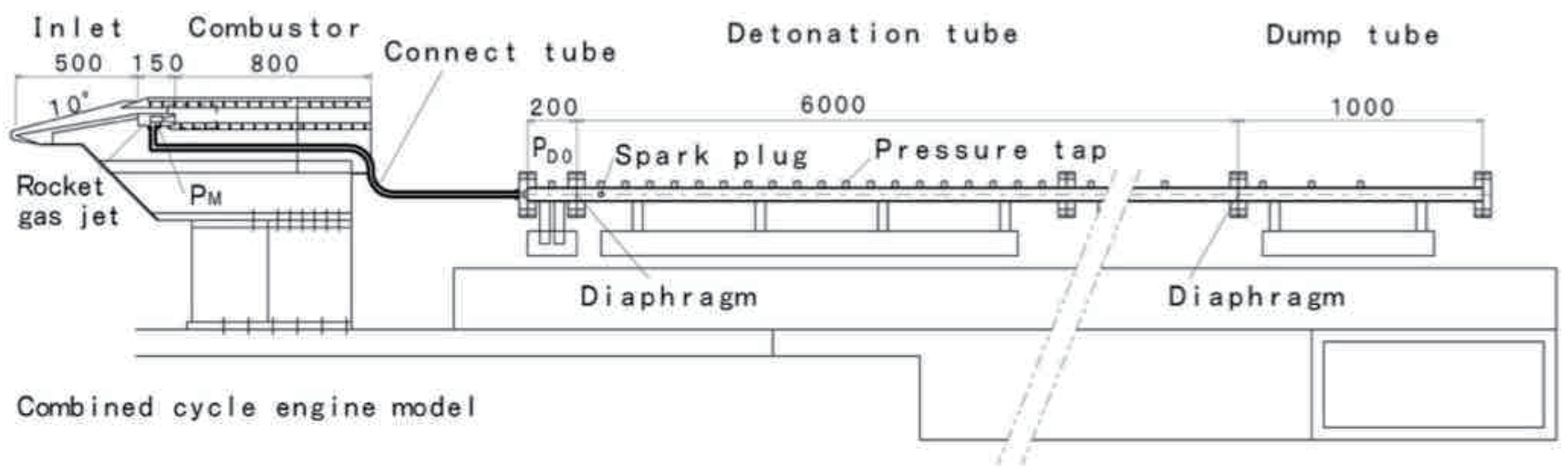

Fig. 3. Schematics of experimental setup.

ramp-side wall.

The rocket chambers were connected to the detonation tube to generate fuel-rich rocket exhaust gas. Figure 3 shows the set up of the facility test apparatus. The detonation tube was filled with hydrogen / oxygen mixture, and a spark-plug was used to initiate detonation traveled away from the rocket chambers. Once the detonation wave was initiated, resulting high-pressure combustion gas ruptured a diaphragm and flew into the rocket chambers. Table 2 shows the typical operation conditions of the detonation tube, and resulting rocket exhaust conditions. The equivalence ratio of residual fuel in the rocket exhaust $\left(\phi_{\mathrm{r}}\right)$ was defined against airflow.

As mentioned above, more auxiliary ramjet-fuel would

Table 2. Detonation tube conditions.

\begin{tabular}{cccc}
\hline Detonation $\begin{array}{c}\text { tube } \\
\text { premixed } \\
\text { gas }\end{array}$ & Equivalence ratio & 1.0 & 1.5 \\
\cline { 2 - 4 } $\begin{array}{c}\text { gatial Pressure } \\
\text { condition }\end{array}$ & $0.5 \mathrm{MPa}$ & $0.5 \mathrm{MPa}$ \\
\hline
\end{tabular}

results in lesser thrust production. In the present study, however, at least half of ramjet-fuel was through the auxiliary injection to attain ignition of auxiliary ramjet-fuel. Specific impulse of the rocket exhaust calculated with a one-dimensional code ${ }^{9)}$ was $418 \mathrm{sec}$. and $378 \mathrm{sec}$. for the $\phi_{\mathrm{r}}=0.5$ and 1.0 (whole ramjet-fuel through the rocket chamber) cases, respectively. Thus, the auxiliary injection could result in a sizable penalty in Isp if combustion of the ramjet-fuel could not be attained.

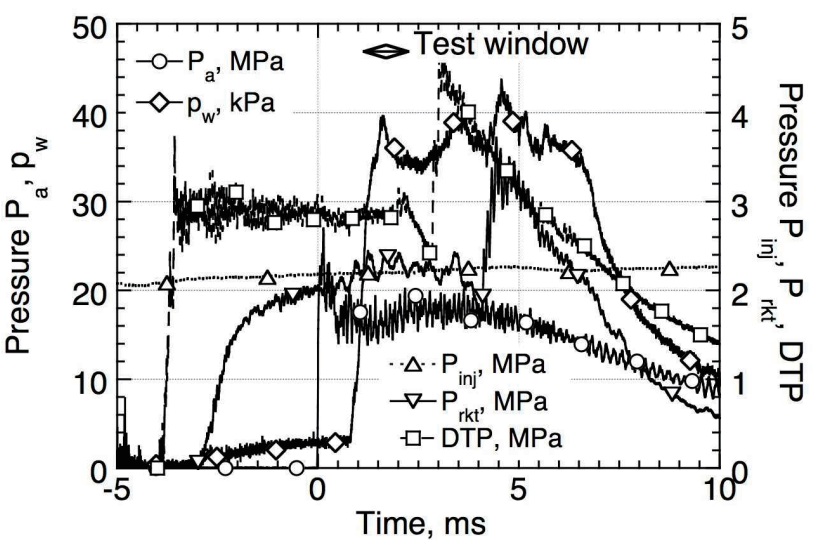

Fig. 4. Time histories of freestream reservoir and rocket chamber pressure.
The technical challenge was to synchronize the tunnel operation and the detonation tube operation. Based on quasi-one dimensional analyses, the time lag from the initiation to the steady gas flow supply was evaluated. A sensor to detect the shock wave arrival in HIEST triggered a timer, and the detonation tube was initiated with the evaluated time lag. Figure 4 shows the time histories of freestream reservoir pressure (i.e., pressure at the end of the shock tube, P0) and rocket chamber pressure. Steady rocket chamber pressure was observed during the test window with steady freestream reservoir pressure. Thus, the shock tunnel (HIEST) and the detonation tube operations were nicely synchronized.

As for the measurement, wall pressure distributions were measured on the ramp wall and the cowl wall within the model's symmetry plane by high frequency pressure transducer (Kulite model XCL-100; range $14.7 \mathrm{kPa}$, error $0.1 \%$ full scale). The measured wall pressure was normalized with the reservoir pressure to mitigate run-to-run deviation.

\subsection{Test results}

As a diverging combustor was applied in the present study, it was expected that attaining combustion was more difficult than in the last study with constant area combustor. Thus, only the case with auxiliary injection $\left(\phi_{\mathrm{i}}=0.5\right.$ and $\left.\phi_{\mathrm{r}}=0.5\right)$ was conducted in the present study.

Figure 5 shows the wall pressure distributions on the ramp in the reactive (with open symbols) and non-reactive (with closed symbols) cases. Either ramp-side injection or cowl-side injection was applied for auxiliary injection.

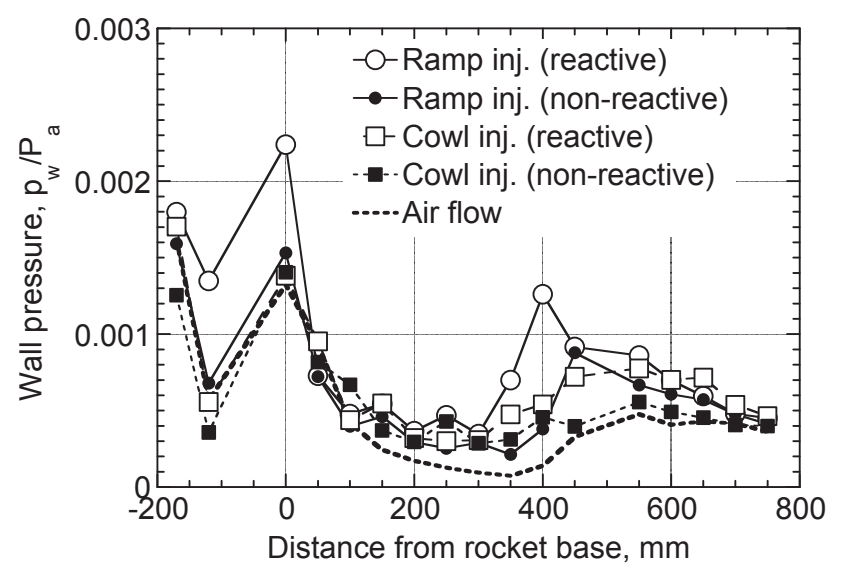

Fig. 5. Wall pressure distributions. 
Pressure distribution in the case with airflow alone is also shown in the figure.

With the ramp-injection, pressure-rise due to ramjet-fuel combustion was attained even around the auxiliary injector. Higher pressure-level in the reactive case than in the non-reactive case was attained throughout the ramjet-combustor. At $X=400 \mathrm{~mm}$, peak pressure location was shifted upstream about $50 \mathrm{~mm}$, as combustion upstream changed the shock wave pattern. ${ }^{10)}$ Thus, it was difficult to assess combustion efficiency directly from change in pressure at identical location. Thus, shock locations were so adjusted to calculate pressure-rise due to combustion. The resulting combustion efficiency will be shown shortly.

With the cowl-injection, no sizable pressure-rise due to combustion was attained until $\mathrm{X}=350 \mathrm{~mm}$. Thus, combustion of auxiliary fuel could not be attained around the injector. A thinner boundary layer on the cowl wall in comparison to that on the ramp wall resulted in insufficient size of the recirculation region upstream of the fuel jet ${ }^{11)}$ to attain self-ignition. After ignition delay for $350 \mathrm{~mm}$, combustion of the auxiliary fuel occurred.

Based on the adjusted pressure distributions, pressure-rise was evaluated and local combustion efficiency was calculated by using one-dimensional method. Figure 6 shows the results. Even with the adjustment, it was difficult to quantify these three dimensional effects such as shock pattern deviation, so that local combustion efficiency scattered widely. Combustion efficiency in supersonic flow was said to vary logarithmically with distance from the injector, so that the variation was evaluated by using the least root-mean square method upon the scattered data. This gradual increase showed that combustion was rate-controlled by mixing in the case with the auxiliary injection. As shown in the figure, combustion efficiency with the ramp-side injector was sizably higher than that with the cowl-side injector, even at downstream portion of the ramjetcombustor where pressure-rise was observed in the case with the cowl-side injection

At the ramjet-combustor exit, combustion efficiency was estimated to be $40 \%$ with the ramp-side injection and

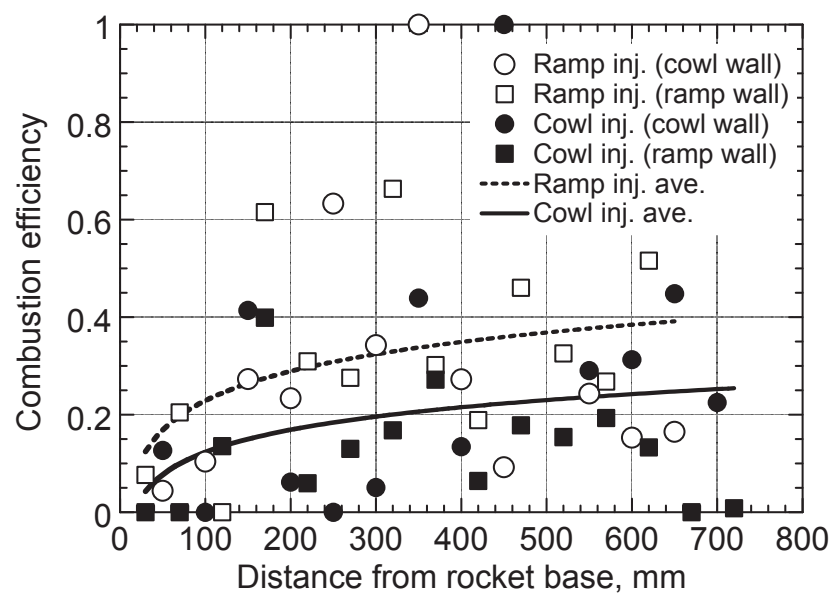

Fig. 6. Combustion efficiency distribution.
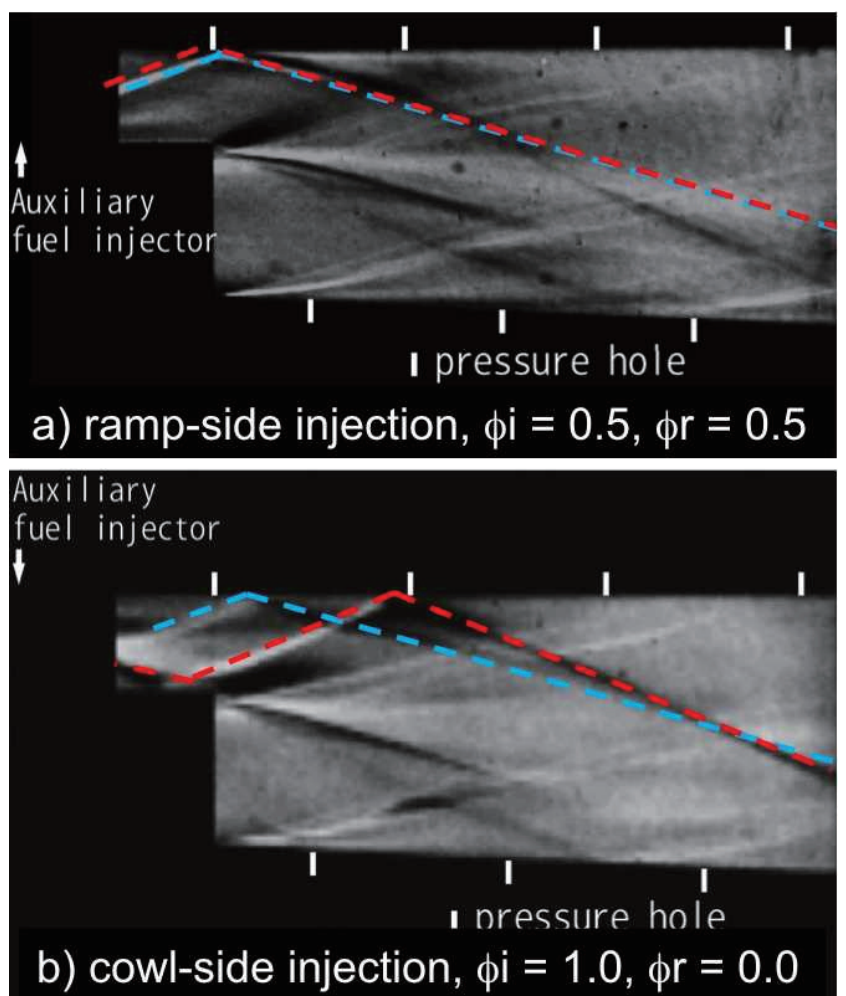

Fig. 7. Schlieren image with a) ramp-side injection (upper) and b) cowl-side injection. (lower)

$25 \%$ with the cowl-side injection. In Ref. 10 , it was reported that combustion efficiency of the normally injected fuel into supersonic combustor under M12 condition was $60 \%$ at most. Using the similar injection scheme, same combustion efficiency level was expected, so that $30 \%$ combustion efficiency for total ramjet-fuel was expected with combustion of auxiliary-fuel. Thus, in the case with the ramp-side injection, combustion of ramjet-fuel within the rocket exhaust should take place, with expected combustion efficiency of $20 \%$ (i.e., (40\% $30 \%$ ) / 0.5). On the other hand, in the case with the cowl injection, combustion of only auxiliary fuel was expected with rather long ignition delay. Combustion of the auxiliary fuel was expected to bring pressure-rise, resulting in higher pressure, higher temperature, and lower velocity, and to shorten the ignition delay of the fuel within the rocket exhaust. Thus, in the cowl-side injection case, ignition devices such as cavity flame holder ${ }^{12)}$ should be installed.

Figures $7 \mathrm{a}$ and $7 \mathrm{~b}$ show the Schlieren image around the rocket base in the case with the ramp-side injection and in the case with the cowl-side injection, respectively. Note that the cowl-side injection flow rate (in equivalence ratio) in Fig. 7b was different from those in Fig. 5, but the feature of the flow field was almost identical (i.e., no sizable pressure-rise in the isolator). In both cases, cowl shock wave (marked with blue line) and bow shock wave (marked with red line) due to auxiliary injection and combustion were visible. These two shock waves merged together to form further intensive shock wave in the case with the ramp-injection, while they crossed with 
each other in the case with the cowl-injection. In the former case, further combustion enhancement of the ramjet-fuel within the rocket exhaust was expected. Incidence of shock wave upon the shear mixing layer was reported to enhance mixing, ${ }^{13)}$ so that mixing study was necessitated to understand combustion phenomena within the ramjet-combustor. However, too short testing time of HIEST made quantitative assessment of mixing process difficult, so that mixing tests were conducted in a blow-down type facility.

\section{Mixing Tests}

Mixing tests were conducted as complimentary to the HIEST tests. Major parameters ${ }^{14)}$ of shear mixing were matched to the engine tests case, and gas sampling was carried out. Table 3 compares the mixing parameters in the engine tests and the mixing tests.

As shown in the table, both static pressure ratio and convective velocity to airflow velocity ratio were almost matched. Note that the rocket exhaust and the airflow were at almost pressure-matched condition. The shear growth parameter, however, could not be matched nicely based on the flow state at the rocket base location. Both the airflow and the rocket exhaust expanded to solve the pressure mismatch and to fill the vacant cross section caused by the large rocket base area, so that the shear growth ratio was rather matched when calculated based on the flow states after the isentropic expansion of two flow tubes filling the entire cross-section at balanced pressure. Thus, the mixing tests were thought to sufficiently compliment the engine tests.

Table 3. Mixing parameters. $\left(\phi_{\mathrm{i}}=0.5\right.$ and $\left.\phi_{\mathrm{r}}=0.5\right)$

\begin{tabular}{|c|c|c|}
\hline \multirow{4}{*}{$\begin{array}{c}\text { Engine } \\
\text { tests }\end{array}$} & $\mathrm{p}_{\mathrm{r}} / \mathrm{p}_{\mathrm{a}}$ & 0.88 \\
\hline & $\mathrm{Uc} / \mathrm{U}_{\mathrm{a}}\left(\mathrm{Eq} \cdot 3^{14)}\right)$ & 1.12 \\
\hline & Shear growth (Eq. $5^{14)}$ ) & 0.029 \\
\hline & Shear growth after exp. & 0.026 \\
\hline \multirow{4}{*}{$\begin{array}{c}\text { Mixing } \\
\text { tests }\end{array}$} & $\mathrm{p}_{\mathrm{r}} / \mathrm{p}_{\mathrm{a}}$ & 0.88 \\
\hline & $\mathrm{Uc} / \mathrm{U}_{\mathrm{a}}\left(\mathrm{Eq} \cdot 3^{14)}\right)$ & 1.11 \\
\hline & Shear growth (Eq. $\left.5^{14)}\right)$ & 0.025 \\
\hline & Shear growth after exp. & 0.027 \\
\hline
\end{tabular}

\subsection{Test apparatus and measurements}

Experiments were carried out in the M2.5 blow-down wind tunnel with a scaled flow-pass to the engine model at HIEST with minor differences (using straight duct instead of diverging duct in the HIEST model, and different airflow aspect ratio in isolator). Figure 8 shows schematics of the test apparatus. Note that ramp wall was on upper side in this apparatus.

Room temperature air was accelerated to Mach 2.5 through a rectangular nozzle. Rocket exhaust was simulated with room temperature nitrogen. Rocket nozzle Mach number was 4 to attain matched airflow / rocket exhaust velocity ratio to that in HIEST tests. Rocket chamber pressure was so selected to attain matched airflow / rocket exhaust static pressure ratio.

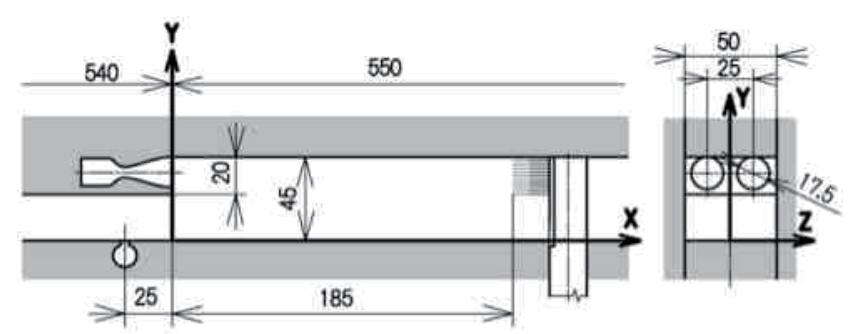

Cowl wall to simulate shock wave incidence

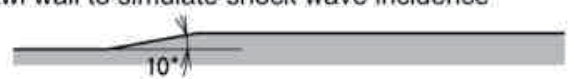

Fig. 8. Mixing study apparatus.

Helium was injected to simulate auxiliary injection. The injector orifice was so selected that the resulting jet penetration height ${ }^{15}$ ) / the base height (as a typical length scale) ratio was matched with that in HIEST case at identical jet to airflow dynamic pressure ratio.

In some cases, a wedged cowl wall was installed to simulate shock wave incidence on the shear layer

Gas sampling was carried out at $185 \mathrm{~mm}$ (9.3*base height, corresponding to $\mathrm{X}=370 \mathrm{~mm}$ in HIEST model) downstream of the using gas chromatography. As for the data reduction, the following procedure was taken. For the auxiliary fuel mole fraction,

1) Measured local injectant $(\mathrm{He})$ to air mole ratio was normalized with overall injectant flow to airflow mole ratio.

2) Nominal equivalence ratio of auxiliary fuel in the HIEST case was multiplied to attain 'equivalent' equivalence ratio in the mixing tests.

For the ramjet-fuel within the rocket exhaust,

1) Local rocket nitrogen to air mole ratio was deduced from measured composition.

2) Local rocket gas to air mole ratio was normalized with overall rocket to airflow mole flow ratio.

3) Nominal equivalence ratio of ramjet-fuel in the rocket plume in the HIEST case was multiplied to attain 'equivalent' equivalence ratio in the mixing tests.

In both cases, mass flux was calculated based on Pitot pressure and wall pressure data as well as the measured composition. Then the mass-flux-weighted local fuel fraction was integrated over the measured cross-section to attain average over the cross- section.

Wall pressure distributions were measured by using a scanning type sensor (scannivalve, range; $350 \mathrm{kPa}$, error; $\pm 1.4 \mathrm{kPa}$ ), and Schlieren images were taken.

\subsection{Test results}

First, mixing between the airflow and the rocket exhaust (containing all ramjet-fuel) was investigated. Figure 9 shows the 'equivalent' equivalence ratio contours without shock wave incidence (right side of the figure) and with the shock wave incidence (left side of the figure). In both cases, $\phi_{\mathrm{r}}=1$ condition was simulated. In the case with the shock wave incidence, the shear layer was bended toward the ramp side, and was broadened in comparison to that without shock wave incidence. The resulting mixing efficiency was $12 \%$ and $29 \%$, respectively, the latter being close to the evaluated combustion efficiency of ramjet-fuel within the rocket 
exhaust in HIEST tests. Thus, with shock wave incidence, mixing process within the HIEST combustor could reasonably simulated.

Figure 10 shows Schlieren images in the case without shock wave incidence and in the case with the shock wave incidence. Shear layer was bended toward the ramp wall by the incidence, but the thickness of the shear layer did not change. On the other hand, shear layer was broadened sizably at expansion wave incidence. Similar phenomena was observed within supersonic combustor, ${ }^{16)}$ incidence of expansion fan upon the jet - airflow mixing layer being reported to enhance mixing.

Next, effects of auxiliary injection on overall mixing characteristics were evaluated. For clarity, the wedged cowl wall was removed. Figure 11 a shows the 'equivalent' equivalence ratio contours with the ramp-side injection (left side of the figure) at simulated ' $\mathrm{f}_{\mathrm{i}}=0.5$ ' + ' $\mathrm{f}_{\mathrm{r}}=0.5$ ' condition, and without auxiliary injection (right side of the figure) as reference (with simulated $f_{r}=1$ condition). The auxiliary fuel was entrained into the shear layer, and the resulting mixing layer was almost identical to that without auxiliary injection. The resulting mixing efficiency of the total ramjet fuel was $14 \%$, and that of the ramjet fuel within the rocket exhaust was $9 \%$. In the case without auxiliary injection, the

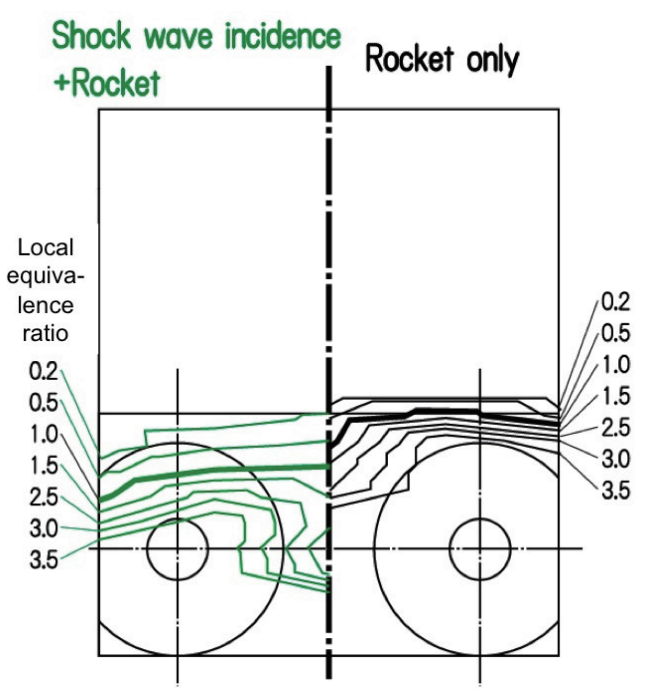

Fig. 9. Equivalent euivalence ratio contour w/o auxiliry injection.
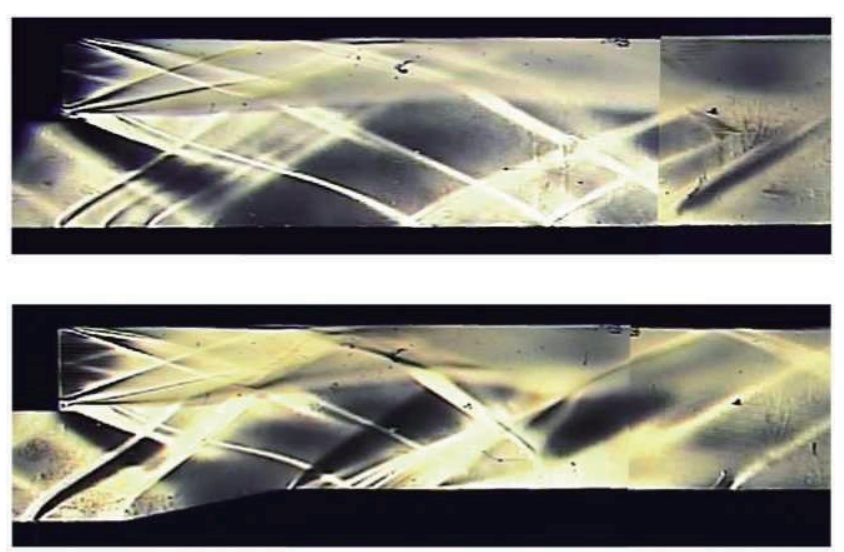

Fig. 10. Schlieren image in case without shock wave incidence (upper) and with shock wave incidence. (lower) shear layer was penetrated the base area, so that streamwise vortex pair was formed between the rocket plume. However, this vortex pair was not obvious in the case without auxiliary injection, probably because the jet - airflow interaction caused different vortex pair in opposite rotational direction to those between the rocket exhaust, so that those pairs were weakened through interaction with each other, and mixing efficiency of the ramjet-fuel within the rocket exhaust became lower than that in the reference case.

Figure $11 \mathrm{~b}$ shows the 'equivalent' equivalence ratio contours with the cowl-side injection (left side of the figure) at simulated ' $\phi_{\mathrm{i}}=0.5$ ' $+{ }^{\prime} \phi_{\mathrm{r}}=0.5$ ' condition, and without auxiliary injection (right side of the figure) as reference (with simulated $\phi_{\mathrm{r}}=1$ condition). Bow shock wave and following expansion fan interacted with the shear layer, so that the shape of the shear layer cross-section was somehow different from that without auxiliary injection. The auxiliary fuel and rocket exhaust was utterly separated and caused no interaction with each other except the wave - shear interaction. The rapid mixing of the auxiliary fuel increased overall mixing efficiency to $31 \%$, with mixing efficiency of the ramjet
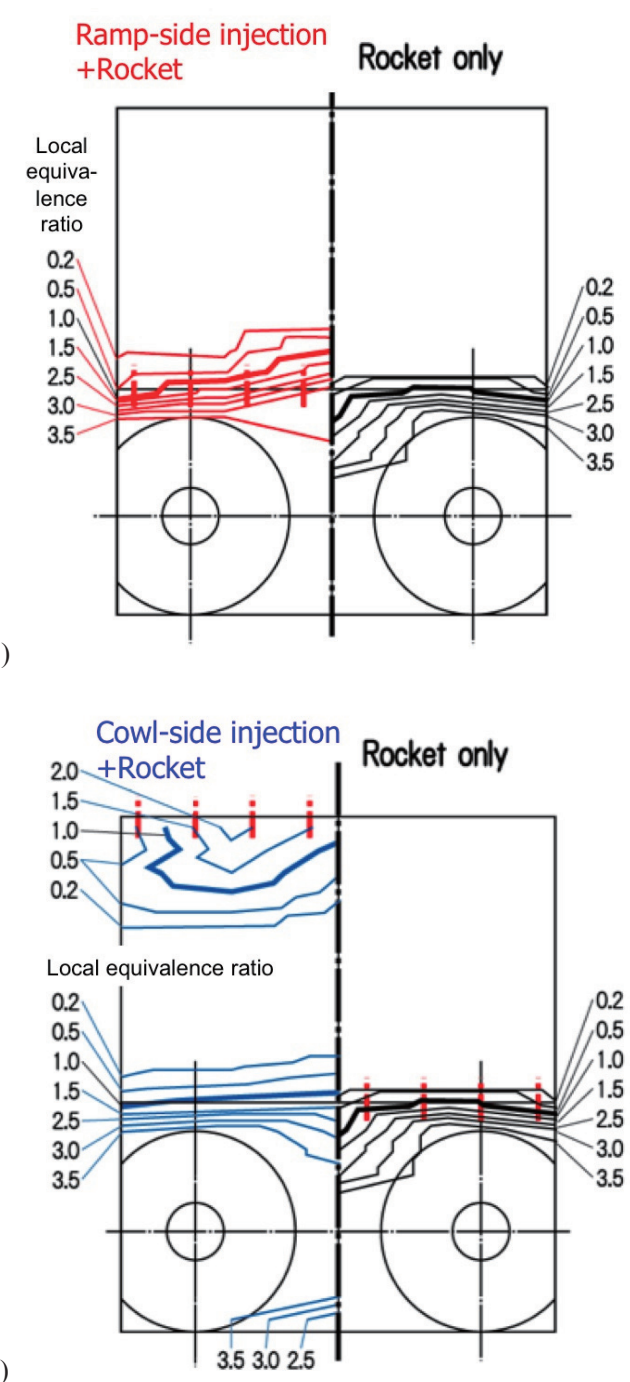

Fig. 11. Equivalent euivalence ratio contour with a) ramp-side injection and b) cowl-side injection. 
fuel within the rocket exhaust was $11 \%$, almost identical to that without auxiliary injection.

From the mentioned findings, it was suggested that 1) cowl-side injection was more beneficial to attain pressure-rise with smaller auxiliary fuel flow rate in terms of mixing characteristics, and 2) Ignition devices such as cavity should be installed to attain combustion of auxiliary fuel through the cowl-side injection.

\section{Conclusions}

An RBCC model using embedded high output rocket chambers for fuel injection to the RBCC's ramjet combustor was tested at Mach 11 flight conditions at the high enthalpy shock tunnel facility, with auxiliary injector added, and following conclusions were derived:

1) With auxiliary injection, combustion of fuel within the rocket exhaust was attained, but combustion efficiency was rather limited (20\% of the fuel in the exhaust).

2) Injection surface was sizable effects of combustion augmentation by auxiliary injection.

Complementary mixing experiments revealed that:

3) Ramp-side auxiliary injection was hazardous as it might suppress airflow-rocket exhaust mixing. As results, cowl-side auxiliary injection with ignition devices was proposed.

\section{References}

1) Kanda, T. and Kudo, K.: Conceptual Study of a CombinedCycle Engine for an Aerospace Plane, J. Propulsion and Power, 19 (2003), pp. 859-867.

2) Siebenhaar, A. and Bulman, M. J.: The Strutjet Engine: The Overlooked Option for Space Launch AIAA paper 95-3124, 1995.

3) Ketchum, A. et. al.: Summary of Rocketdyne Engine A5 Rocket Based Combined Cycle Testing, NAS8-40894, 1998.

4) Whitlow Jr, W. et. al.: Innovative Airbreathing Propulsion Concepts for Access to Space, NASA/TM-2001-210564, 2001.

5) Perkins, H. D. et. al.: Mach 5 to 7 RBCC Propulsion System Testing at NASA-LERC HTF, AIAA paper 97-0565, 1997.

6) Tomioka, S. et. al.: Combustion Enhancement in ScramjetOperation of a RBCC Engine, Trans. JSASS Space Tech. Japan, 9, ists28 (2011), pp. Pc_1-Pc_6.

7) Itoh, K. et. al.: Hypersonic Aerothermodynamic and Scramjet Research Using High Enthalpy Shock Tunnel, Shock Waves, 12 (2002), pp. 93-88

8) Takegoshi, M. et. al:: Mach 8 Flight condition Tests of Rocket-Ramjet Combined-Cycle Engine Model, AIAA paper 2013-3668, 2013.

9) Gordon, S. and Mcbride, B. J.: Computer program for calculation of complex chemical equilibrium compositions and applications. Part 1: Analysis, NASA-RP-1311, 1994.

10) Takahashi, M. et. al.: Performance Characteristics of a Scramjet Engine at Mach 10 to 15 Flight Condition, AIAA paper 2005-3350, 2005 .

11) P. W. Huber, C. J. et. al.: Criteria for self-ignition of supersonic hydrogen-air mixtures NASA TP-1457, 1979.

12) $\mathrm{Yu}, \mathrm{K}$. et. al.: Effect of Flame-Holding Cavities on Supersonic Combustion Performance, AIAA paper 99-2638, 1999.

13) $\mathrm{Lu}, \mathrm{P} . \mathrm{J}$. and $\mathrm{Wu}, \mathrm{K}$. C.: On the Shock Enhancement of Confined Supersonic Mixing Flows, Physics of Fluids A, 3 (1991), pp. 3046-3062.
14) Papamoschou, D.: Model for Entropy Production and Pressure Variation in Confined Turbulent Mixing, AIAA J., 31 (1993), pp. 1643-1650.

15) Cohen, L. S. et. al.: Penetration and Mixing of Multiple Gas Jets Subjected to a Cross Flow, AIAA J., 9 (1971), pp. 718-724.

16) Yokota, K. and Kaji, S.: Two and Three Dimensional Study on Supersonic Flow and Mixing Fields with Hydrogen Injection, AlAA paper 1995-6024, 1995. 